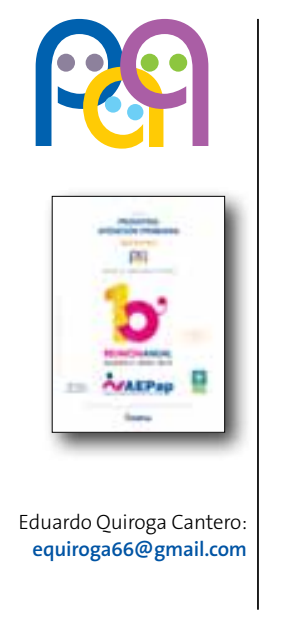

\section{Cuidados paliativos: qué debe saber un pediatra de Atención Primaria}

\section{Foro profesional}

\section{E. Quiroga Cantero}

Coordinador del Grupo de Apoyo de Cuidados Paliativos Pediátricos. Hospital Infantil Virgen del Rocío, Sevilla. España.
Los Cuidados Paliativos Pediátricos (CCPP) pretenden conseguir la atención integral del enfermo terminal y de su familia, tanto en aspectos físicos como emocionales, sociales y espirituales, promocionando la autonomía y respetando la dignidad del enfermo mediante una atención continuada e individualizada.

El primer paso sería saber identificar qué niños de los que atendemos en Atención Primaria o Atención Especializada serían subsidiarios de CCPP, evitando el concepto erróneo de pensar que serían solo aquellos niños que padecen enfermedades graves y cuya muerte es inminente. Por el contrario, la mayoría de los niños de este ámbito padecen enfermedades neurológicas o metabólicas crónicas que pueden sobrevivir años, aunque la mayoría de ellos no llegarán a la edad adulta.

A continuación desglosaremos las diferentes enfermedades englobados en los Grupos de la IMPACCT que recoge los niños subsidiarios de CCPP:

- Grupo 1: aquellos niños con enfermedades graves pero potencialmente curables y donde el tratamiento puede fallar. Por ejemplo, cáncer Infantil.

- Grupo 2: enfermedades donde la muerte prematura es inevitable y donde puede haber lar- gos periodos de tratamiento intensivo dirigido a prolongar la vida, pero en las que el paciente puede participar en actividades normales de la misma. Por ejemplo, fibrosis quística.

- Grupo 3: enfermedades progresivas sin opciones de tratamiento curativo, donde el tratamiento es paliativo y se puede extender durante largo tiempo. Por ejemplo, distrofia muscular.

- Grupo 4: enfermedades irreversibles pero no progresivas que causan gran discapacidad y alta susceptibilidad a complicaciones de salud y muerte prematura. Por ejemplo, parálisis cerebral.

Una vez identificados qué niños serían subsidiarios de CCPP, deberíamos responder estas cuestiones:

- ¿Qué necesitan los niños con enfermedades no curables o terminales?

- Unidad de Cuidados Paliativos Pediátricos: ¿quienes somos?

- ¿Qué es la Atención Continuada?

- ¿Dónde se pueden tratar los niños con enfermedades no curables?

- Ventajas e inconvenientes del domicilio. 


\section{ATENCIÓN EN EL DOMICILIO}

La atención en el domicilio debe ser realizada por profesionales coordinados cuyo objetivo principal sea conseguir el mejor cuidado de su niño y de su entorno familiar. Esta atención en el domicilio tiene sus ventajas e inconvenientes:

\section{Ventajas}

- Entorno conocido.

- Mayor comodidad del paciente y la familia (flexibilidad horarios, comidas caseras, juguetes y cama propia...).

- Favorece la cohesión y los vínculos familiares.

- Evita los desplazamientos del paciente y familiares al centro sanitario.

- Concentra la asistencia en un grupo reducido de profesionales (continuidad y coherencia de los cuidados).

- Disminuye los gastos para la familia y el sistema sanitario.

\section{Inconvenientes}

- Requiere mayor participación de los familiares en la valoración y aplicación de los cuidados.

- Incertidumbre sobre la aparición de nuevos síntomas y miedo a no saber manejarlos.

- Dudas en los familiares sobre la propia capacidad.

- Temor ante lo desconocido y ante la posibilidad de quedar desasistidos.

- Mayor tiempo de respuesta por los profesionales.

- Valorar condiciones habitabilidad del domicilio.

El plan de cuidados en el domicilio es fundamental y no debe ser rígido sino que debe ser flexible, pues la situación del niño y su familia puede cambiar de día a día. Dependiendo de la complejidad de cada caso y del momento evolutivo, los equipos de CCPP adquirirán un protagonismo progresivo, teniendo en cuenta que siempre el Pediatra de Atención Primaria es el médico referente y las decisiones o actuaciones deben consensuarse con él.
Por tanto, depende del nivel de complejidad, puede ser solo el Pediatra de Atención Primaria sea quien preste la ayuda a domicilio junto con la enfermería adecuada y el equipo de CCPP sea un mero consultor o que el trabajo sea realizado de forma conjunta.

\section{EVALUACIÓN INTEGRAL Y PLAN DE CUIDADOS}

\section{Cuestiones a considerar}

- Situación actual del niño y de su familia.

- Información y Toma de decisiones.

- Complicaciones posibles

- Intervenciones a realizar.

Respecto a la situación actual del niño y su familia, tendremos en cuenta la situación sanitaria, social, espiritual y psicológica, y la toma de decisiones conveniente:

\section{- Sanitaria:}

- Problemas actuales (dolor, nutrición, hematológico, neurológico, infeccioso...).

- Tratamiento actual.

- Complicaciones posibles y manejo en casa.

- Vías de administración de la medicación.

- Cuidados especiales: oxígeno, aspirador secreciones..

- Controles: otros especialistas, analíticas...

\section{- Toma de decisiones:}

- Lugar atención/lugar fallecimiento.

- Grado información del niño y capacidad de entendimiento.

- Adecuación de medidas, informe de no resucitación cardiopulmonar (RCP).

\section{- Social:}

- Identificar a los cuidadores.

- Situación laboral y económica.

- Apoyo familiar/social.

- Riesgo social.

- Valorar vivienda. 
- Valorar ayudas sociales.

- Trámites funerarios.

\section{- Psicológica:}

- Estructura y dinámica familiar.

- Afrontamiento del niño y la familia.

- Modelo de comunicación.

- Problemas de relación reales y potenciales.

- Atención al duelo.

\section{- Espiritual:}

- Creencias (religiosas o no).

- Trascendencia, sentido.

- Valores.

\section{CASO CLÍNICO}

Niño de nueve años con parálisis cerebral infantil, que vive en un pueblo de Sevilla, y que es Ilevado frecuentemente al centro de salud e ingresa con asiduidad en el hospital de referencia por reagudizaciones respiratorias, donde también está siendo tratado por el equipo de Neurología Pediátrica.

\section{Antecedentes personales}

- Encefalopatía hipóxico-isquémica neonatal.

- Tetraparesia espástica y retraso psicomotor grave.

- Epilepsia secundaria de difícil control.

- Neumopatía crónica multifactorial: infecciones respiratorias de repetición.

- Déficit visual grave.

- Portador de gastrostomía con Nissen.

- Subluxación de caderas intervenida.

\section{Exploración}

- Buen estado general. Bien nutrido. Deformidades craneofaciales.

- Espasticidad generalizada. Escoliosis, deformidad de caja torácica. Luxación de caderas.

- Microcefalia. Escaso contacto con el medio, reacciona a estímulos dolorosos. Ausencia de movilidad espontánea.
- Auscultación cardiopulmonar: buena ventilación bilateral con roncus. Abdomen blando, con botón de gastrostomía.

\section{Situación actual}

- Viven en un pueblo de Sevilla.

- Primer hijo de padres sanos. Hermano de cuatro años sano.

- Escolarizado en colegio de educación especial.

- Cuidadora principal: madre. Dedicación exclusiva. ¿Padre ausente?

- Nivel socioeconómico medio.

- Padres informados que piden no RCP pero sí tratamiento sintomático.

- Quieren estar en casa y, si llega el momento, que muera allí.

- Apoyo familiar.

- Católicos no practicantes.

\section{Toma de decisiones}

- Evitar ingresos hospitalarios.

- Manejo de complicaciones (respiratorias/convulsiones) intercurrentes.

- Facilitar la cohesión y los vínculos familiares mediante la hospitalización domiciliaria.

- Limitación de las pruebas diagnósticas.

- Establecimiento de objetivos en cada fase de la enfermedad y adecuación de medidas: informe no RCP.

Después de estas valoraciones realizadas por el Pediatra de Atención Primaria, se avisa al equipo de CCPP y se establece un plan de cuidados conjunto, con visitas programadas. El equipo de CCPP contacta a su vez con el neurólogo pediátrico de referencia.

\section{Evolución}

- Neurológico: reagudizaciones coincidiendo con episodios infecciosos, que en ocasiones requieren diazepam rectal.

- Digestivo-nutrición: fórmula polimérica normocalórica con fibra, fraccionada en dos tomas 
diurnas y enteral continua nocturna. Estreñimiento crónico en tratamiento con laxante oral.

- Ortopédico: espasticidad en tratamiento con baclofeno oral.

- Piel: riesgo de úlcera perforante por presión. Control con medidas generales.

- Respiratorio-infeccioso: ocho infecciones respiratorias en seis meses, controladas en el domicilio con antibioterapia oral, oxigenoterapia, aerosolterapia y aspiración de secreciones.

\section{- En el último mes:}

- Secreciones continuas y dependencia de oxígeno para mantener saturaciones en torno al $90 \%$.

- Fiebre y empeoramiento progresivo que no responde a las medidas habituales, precisando administración de morfina oral.

- En las 24 horas previas al fallecimiento, incremento de la dificultad respiratoria con disminución del nivel de conciencia. Perfusión subcutánea de cloruro mórfico y midazolam, retirándose el oxígeno en la fase final para no prolongar la agonía.

\section{BIBLIOGRAFİA RECOMENDADA}

- A guide to the development of children's palliative care services. Association for Children with LifeThreatening or Terminal Conditions and their Families. Bristol, UK: ACT-and The Royal College of Paediatrics and Child Health; 2003.

- Davies B, Sehring SA, Colin Partridge J, Cooper BA, Hughes A, Philp JC, et al. Barriers to Palliative Care for Children: Perceptions of Pediatric Health Care Providers. Pediatrics. 2008;121;282-8.
- Fallece acompañado de su familia, sin dolor y tranquilo.

La familia de este niño fue apoyada por la psicóloga del equipo de CCPP durante el proceso del paciente, en el momento de la muerte y en el duelo posterior.

La trabajadora social del equipo de CCPP resultó de ayuda para resolver los problemas sociales que acompañaban a la familia durante la enfermedad y después de esta.

Desde el punto de vista espiritual, la trascendencia de la muerte del niño para los padres fue la de comprometerse posteriormente con otras familias necesitadas, y agradecidos porque su hijo pudiera haber muerto en paz y en su casa.

\section{CONFLICTO DE INTERESES}

El autor declara no presentar conflictos de intereses en relación con la preparación y publicación de este artículo.

\section{ABREVIATURAS}

CCPP: Cuidados Paliativos Pediátricos - RCP: resucitación cardiopulmonar.
- Hinds PS, Drew D, Oakes L, Fouladi M, Spunt SL, Church $C$, et al. End-of-life care preferences of pediatric patients with cancer. J Clin Oncol. 2005;23:914654.

- IMPaCCT-standards for paediatric palliative care in Europe. Eur J Pall Care. 2007;14:109-14.

- World Health Organization. WHO definition of Palliative Care for Children [en línea] [consultado el 23/10/2006]. Disponible en: www.who.int/cancer/ palliative/definition/en/ 